\title{
DIFFERENTIAL GALOIS THEORY AND NON-INTEGRABILITY OF PLANAR POLYNOMIAL VECTOR FIELDS
}

\author{
PRIMITIVO B. ACOSTA-HUMÁNEZ, J. TOMÁS LÁZARO, JUAN J. MORALES-RUIZ, \\ AND CHARA PANTAZI
}

\begin{abstract}
We study a necessary condition for the integrability of the polynomials vector fields in the plane by means of the differential Galois Theory. More concretely, by means of the variational equations around a particular solution it is obtained a necessary condition for the existence of a rational first integral. The method is systematic starting with the first order variational equation. We illustrate this result with several families of examples. A key point is to check whether a suitable primitive is elementary or not. Using a theorem by Liouville, the problem is equivalent to the existence of a rational solution of a certain first order linear equation, the Risch equation. This is a classical problem studied by Risch in 1969, and the solution is given by the "Risch algorithm". In this way we point out the connection of the non integrablity with some higher transcendent functions, like the error function.
\end{abstract}

\section{INTRODUCTION}

The problem of the integrability of planar vector fields has attracted the attention of many mathematicians during decades. Among different approaches, Galois Theory of linear differential equations has played an important rôle in its understanding, even in the a priori (so far) simpler case of polynomial vector fields (see $[4,24,1]$ and references therein). For instance, the application of differential Galois Theory to variational equations along a given integral curve constitutes a powerful criterium of non-integrability for Hamiltonian systems (see [16]). Extensions of this method for some non Hamiltonian vector fields have been carried out by Ayoul and Zung [2]. They strongly rely on the main result of [18].

Many authors have tackled the problem of integrability from other different points of view. For instance, we refer the reader to the papers [19, 23, 27, 28] and references therein, where necessary conditions on Liouvillian and Elementary integrability are provided.

The aim of this paper is to apply Galois Theory to prove the non-existence of rational first integrals for a kind of planar polynomial vector fields. Indeed, let us consider planar vector fields of the form

$$
X=P \frac{\partial}{\partial x}+Q \frac{\partial}{\partial y},
$$

with $P, Q$ analytic functions in some domain of $\mathbb{C}^{2}$ and assume $\Gamma: y-\varphi(x)=0$ to be an integral curve ${ }^{1}$ of $X$. This is equivalent to say that $\Gamma$ is a solution (a leaf) of the first order

Date: January 26, 2018.

2010 Mathematics Subject Classification. Primary: 12H05. Secondary: 32S65.

${ }^{1}$ Usually it is also referred as an orbit of the ode system $\dot{x}=P, \dot{y}=Q$. In qualitative theory of dynamical systems this is commonly called invariant curve. 
differential equation

$$
y^{\prime}=\frac{Q}{P}=f(x, y),
$$

which defines its associated foliation (orbits of the vector field $X$ ). From now on ' will denote derivative with respect to the spatial variable $x$. The behaviour around the solution $\Gamma$ is usually approached by studying its variational equations. In our case, with respect to equation (2). Precisely, let $\phi(x, y)$ denote the flow of $(2)$. Consider $\left(x_{0}, y_{0}\right)$ a point in $\Gamma$, that is, $y_{0}=\varphi\left(x_{0}\right)$. Note that $\left(x_{0}, y_{0}\right)$ is the initial condition defining $\Gamma: \varphi(x)=\phi\left(x, y_{0}\right)$. We are interested in the variation of the flow $\phi$ respect to the initial condition $y$, around $y=y_{0}$ and keeping $x=x_{0}$ fixed. In other words, in the flow defined by the initial condition $\phi\left(x_{0}, y\right)=y$. This means that we want to compute the Taylor expansion coefficients

$$
\varphi_{k}(x)=\frac{\partial^{k} \phi}{\partial y^{k}}\left(x, y_{0}\right)
$$

for which

$$
\phi(x, y)=\varphi(x)+\frac{\partial \phi}{\partial y}\left(x, y_{0}\right)\left(y-y_{0}\right)+\frac{1}{2} \frac{\partial^{2} \phi}{\partial y^{2}}\left(x, y_{0}\right)\left(y-y_{0}\right)^{2}+\cdots
$$

becomes a solution of the variational equations. Namely, the first two variational equations $\mathrm{VE}_{1}$ and $\mathrm{VE}_{2}$ are explicitly given by

$$
\begin{aligned}
& \varphi_{1}^{\prime}=f_{y}(x, \varphi(x)) \varphi_{1}, \\
& \varphi_{2}^{\prime}=f_{y}(x, \varphi(x)) \varphi_{2}+f_{y y}(x, \varphi(x)) \varphi_{1}^{2},
\end{aligned}
$$

where we introduce the standard notation $f_{y}, f_{y y}, \ldots$ for the corresponding partial derivatives $\partial f / \partial y, \partial^{2} f / \partial y^{2}, \ldots$, respectively. In a similar way higher order variational equations $\mathrm{VE}_{k}$, for $k>2$, can be obtained.

It is well-known (due to their triangular-shape scheme), that variational equations $\mathrm{VE}_{k}$ can be solved recurrently. However, in order to apply the Differential Galois Theory, it is convenient to linearize them. This can be easily done by introducing suitable new variables: for example, if one defines $\chi_{1}=\varphi_{1}^{2}$ and $\chi_{2}=\varphi_{2}$, the second variational $\mathrm{VE}_{2}$ becomes

$$
\begin{aligned}
& \chi_{1}^{\prime}=2 f_{y}(x, \varphi(x)) \chi_{1}, \\
& \chi_{2}^{\prime}=f_{y}(x, \varphi(x)) \chi_{2}+f_{y y}(x, \varphi(x)) \chi_{1} .
\end{aligned}
$$

Linearised variational equation of order $k$ will be denoted by $\mathrm{LVE}_{k}$ (see Appendix C for more details). Now, since $\mathrm{LVE}_{k}$ is a linear system, standard differential Galois Theory can be applied. Let $G_{k}$ stand for the Galois group of $\mathrm{LVE}_{k}$ and $G_{k}^{0}$ for its identity component.

Definition 1.1. A complex analytic vector field $X$ defined over an analytic complex manifold $M$ of dimension $m$ is meromorphically integrable if there exist $X=X_{1}, X_{2}, \ldots, X_{k}$ functionally independent pairwise commuting meromorphic vector fields and $f_{1}, f_{2}, \ldots, f_{l}$ functionally independent meromorphic first integrals of these vector fields, and satisfying that $k+l=m$.

In the particular planar case, meromorphically integrability can come from two possibilities:

(a) either $X_{2}$ is a symmetry vector field of $X_{1}$, that is $\left[X_{1}, X_{2}\right]=0$, or

(b) there exists a first integral $H=f_{1}$ of $X_{1}$. 
It is clear that the fibration defined by the level sets of a first integral $H$ of the vector field (1), $H(x, y)=C$, gives the solution of equation (2).

Then we have the following result:

Theorem A. Consider a planar analytic vector field $X$ as in (1) and $\Gamma$ an integral curve. Then, if $X$ is meromorphically integrable in a neighbourhood of $\Gamma$ it follows that, for any $k \geq 1$, the identity component $G_{k}^{0}$ of the Galois group of the linear variational equation LVE $_{k}$ is abelian.

A very interesting particular case of Theorem $\mathrm{A}$ is when the vector field $X$ is meromorphic at infinity, that is, either is holomorphic or it has a pole at $\infty$. Functions of such type are called meromorphic at the extended complex plane. It is known, by compactification of the complex plane, that these functions are in fact the rational functions. This is exactly the case when one considers vector fields (1) with $P$ and $Q$ polynomials.

Therefore, we say that either the vector field (1) or the associated foliation (2) is rationally integrable by substituting meromorphic by rational in Definition 1.1.

Theorem B. Consider a planar polynomial vector field $X$ as in (1) and let $\Gamma$ be an invariant curve. Assume that the first variational equation $\mathrm{VE}_{1}$ along $\Gamma$ has an irregular singular point at infinity. Then, if $X$ is rationally integrable then, for any $k \geq 1$, the Galois group $G_{k}$ of $\mathrm{LVE}_{k}$ is abelian.

Since we will be concerned with rational integrability, it will be natural in general to assume the invariant curve $\Gamma$ to be algebraic.

Recall that a linear system of differential equations with a pole at the origin can always be written in the form $x^{h} Y^{\prime}(x)=B(x) Y$, where $B(x)$ is an analytic matrix at $x=0$ with $B(0)=B_{0} \neq 0$ and $h \in \mathbb{N}$. It is said that $x=0$ is an irregular singular point of this equation if the solutions of this system exhibit an exponential growth around $x=0$ (see for instance [25]).

If the first variational equation $\mathrm{VE}_{1}$ of a planar vector field has an irregular singular point at $x=0$, it can be written as

$$
\varphi_{1}^{\prime}=\frac{1}{h} b(x) \varphi_{1}
$$

being $h>1$ an integer and $b(x)$ analytic at $x=0$ with $b(0) \neq 0$. Furthermore, if the first variational equation $\mathrm{VE}_{1}$ has an irregular singular point then all the following variational equations have the same irregular singular point. This is clear since its exponential behaviour only depends on the first variational equation and the solution of this equation appears in all of the following variational equations ([18]). Thus the Galois group $G_{k}$ of any variational equation is connected and, therefore, it coincides with its identity component, that is $G_{k}=G_{k}^{0}$. A proof of this fact will be carried out in Proposition 3.4. Moreover, explicit representations of the Galois groups $G_{2}$ and $G_{3}$ in the presence of irregular singular points will be provided.

Notice that Theorem B is a necessary condition for rational integrability of a planar vector field whereas Theorem A is a necessary condition for the more general meromorphic integrability of planar analytic vector fields. Besides, the result of Theorem B is true under the additional condition that the first variational equation has an irregular point at infinity.

Theorem A (and B) is commonly applied to prove non-integrability results: if for some $k$ the Galois group $G_{k}^{0}\left(G_{k}\right.$ for Theorem B) is not abelian then the polynomial vector field 
$X$ is not meromorphically (rationally) integrable. Its application is based on the following procedure, similar to the one used for Hamiltonian systems. In the case of Theorem A:

1) To obtain an invariant curve $\Gamma: y-\varphi(x)=0$, of the vector field $X$.

2) To compute $G_{k}$ and to check if the identity component $G_{k}^{0}$ is not abelian (for $k \geq 2$ ).

3) If for some $k, G_{k}^{0}$ is not abelian, then the vector field $X$ is not meromorphically integrable.

4) If for all $k$, one has that $G_{k}^{0}$ is abelian, then the method cannot decide.

Analogously, in the case of Theorem B, the procedure reads:

1') Same as 1) above.

2') To compute $G_{k}$ and to check if it is not abelian (again, for $k \geq 2$ ).

3') If for some $k$, we have that $G_{k}$ is not abelian, then the vector field $X$ is not rationally integrable.

4') If for all $k, G_{k}$ is abelian, then the method cannot decide.

It is important to point out that the so-called Risch algorithm (and its homonymous equation) [20] provides a systematic way to analyse condition 2'). See Section 4 for more details on it.

One of the scopes of this paper is also to use a suitable version of Risch-Kaltofen algorithm (see [11]) to detect non-integrability of planar polynomial vector fields under the hypotheses of Theorem B.

Remark 1.2. It is also possible to obtain interesting results for regular singular points from Theorem A. They are related to the non abelianity of the holonomy of the vector field around the curve $\Gamma$. For this kind of singular points the holonomy of the associated foliation is given, at some order, by the monodromy of the variational equation (see, for instance, [8]); and the monodromy group is Zariski dense in the Galois group. However, in this regular singular case the Galois group $G_{1}$ may be non connected (i.e., it can be given by the group of roots of unity, see [4, Example 6.15]). Hence it is necessary to check this possibility and when we fall in it we have $G_{k}^{0} \neq G_{k}$. Then one should study the non commutativity of the group $G_{k}^{0}$. This approach is not pursued in this paper.

Remark 1.3. Like it happens for Hamiltonian systems, the Achilles' heel of the above procedure is step 1). The key point of our approach is that, for generic polynomial vector fields on the plane, the line at infinity is always an invariant curve $\Gamma$ of the system (see [10]). In the real section of $\mathbb{C}^{2}$, this is connected with the Poincaré compactification (see, for instance, [7, Chapter V] and references therein).

Henceforth it will be assumed some knowledge on Galois Theory of linear differential equations, the so-called Picard-Vessiot Theory (we refer the reader to [4, 24, 13], and references therein, for classical monographs). See also [17]. To our knowledge, this is the first time that the method of the variational equations is applied to study the non-integrability of planar polynomial vector fields.

The rest of the paper is structured as follows: Section 2 contains the proof of Theorems A and $\mathrm{B}$ above. Section 3 is devoted to the analysis of the Galois group of the variational equations, where conditions ( $\mathrm{H} 1)$ and ( $\mathrm{Hk})$ will be introduced. These hypotheses will play 
an essential rôle along the paper. The main result of this paper (see Theorem 3.5), is stated as follows:

Main Theorem. Under the assumptions (H1) and (Hk) (for some $k \geq 2$ ) it follows that the polynomial vector field $X$ (or its corresponding foliation) is not rationally integrable.

In Section 4, assumption (Hk) is related to the Risch differential equation. Variational equations around the line at infinity are considered in Section 5. We provide examples of quite general families and perform an explicit ad-hoc version of the Risch-Kaltofen algorithm in Section 6. A conjecture extending the class of first integrals considered in this paper is presented in Section 7. For the completeness of the work, Appendices on some MAPLE computations, Galois correspondence and third order variational equations have been included.

\section{Proof of Theorems A And B}

In this Section we prove Theorem A. The proof of Theorem B follows in a similar way but in a rational context. The proof is mainly based on two well-known results: (i) MoralesRamis-Simó Theorem and (ii) Ayoul-Zung Theorem. Indeed,

Theorem 2.1 ([18, 17]). Assume that a complex analytic Hamiltonian system is meromorphically integrable in a neighbourhood of an integral curve $\Gamma$. Then the identity components $G_{k}^{0}, k \geq 1$, of the Galois groups of the linear variational equations, $\mathrm{LVE}_{k}$, along $\Gamma$ are abelian.

Here integrability of a Hamiltonian system means that it is integrable in the Liouville's sense: existence of a maximal number of functionally independent meromorphic first integrals in involution.

We stress that Theorem 2.1 has sense for Hamiltonian systems with more than one degrees of freedom. One degree of freedom Hamiltonian systems are trivially integrable.

There are several variants of Theorem 2.1, depending on the nature of the singularities of the first integrals. If the variational equations have irregular singular points at infinity in the phase space then one can compactify it. So, the obstructions for the existence of (meromorphic) first integrals appear only at the infinity. That is,

Theorem 2.2 ([18]). Consider a complex analytic Hamiltonian vector field. Assume that the first variational equation $\mathrm{VE}_{1}$ has an irregular singular point at infinity and that for some $k$, $G_{k}^{0}$ is non abelian. Then the Hamiltonian system is not integrable by means of rational first integrals.

Although the polynomial context is the most common, there are examples in Celestial Mechanics (see also [18]) where Theorem 2.2 has been applied to Hamiltonian non-polynomial vector fields. Recall that in the Hamiltonian framework, the necessary number of first integrals is equal to the half of the dimension of the phase space.

The problem is that, in general, equation (2) does not define a Hamiltonian system, so the associated vector field

$$
Y=\frac{\partial}{\partial x}+f(x, y) \frac{\partial}{\partial y}
$$

is not Hamiltonian on the phase space $(x, y)$. It is worth noticing that the first integrals of the rational vector field (6) are the same as the first integrals of the initial polynomial vector field (1), since for any $H(x, y)$ one has that $X(H)=P Y(H)$. Thus, although the 
vector field $Y$ is not Hamiltonian, it is possible to construct a Hamiltonian system over the cotangent fiber bundle $T^{*} \mathbb{P}_{2}$ (called the cotangent lift of $\left.Y\right)^{2}$ and apply the previous argument on this new Hamiltonian system. Ayoul and Zung considered this case in [2] and obtained the following more general result:

Theorem 2.3 ([2]). Assume that a complex meromorphic vector field $Y$ is meromorphically integrable in a neighbourhood of an integral curve $\Gamma$. Then, for any $k \geq 1$, the identity component $G_{k}^{0}$ of the Galois group of the linear variational equation $\mathrm{LVE}_{k}$ along $\Gamma$ is abelian.

The proof of this theorem is based essentially on the following two facts:

- The Galois group of the variational equation of the system $Y$ is the same as the Galois group of the variational equations of the lifted system.

- The application of Theorem 2.1, which ensures that integrability of $Y$ implies integrability of the lifted system (in the Liouville sense).

Hence, Theorem 2.3 undergoes the same variants as Theorem 2.1. In particular, for variational equations with irregular singular point at the infinity we have obstructions to the existence of rational first integrals, i.e. mutatis mutandis, Theorem 2.2 is also valid here. This concludes the proof of Theorems A and B.

We point out that although Theorems $\mathrm{A}$ and $\mathrm{B}$ are an almost obvious consequence of Theorems 2.1, 2.2 and 2.3, we state them as independent theorems, because the variant of Theorem 2.2 (ie, the existence of an irregular singular points at infinity) was not explicit considered in the reference [2].

Remark 2.4. An alternative proof of these theorems can certainly be done using some relevant results by Casale, which connect Theorems A and B with Malgrange's approach to Galois Theory of nonlinear differential equations [3].

\section{The Galois group of the variational EQUation}

Let us focus our attention in the rational context. Indeed, assume that our vector field is given in the form

$$
X=P \frac{\partial}{\partial x}+Q \frac{\partial}{\partial y}
$$

with $P, Q$ polynomials or, in other words, having a foliation $y^{\prime}=Q / P=f(x, y)$ with rational $f(x, y)$. Let us also suppose that our invariant curve $\Gamma: y-\varphi(x)=0$ is rational. Then the variational equation (4) reads

$$
\begin{aligned}
& \chi_{1}^{\prime}=2 \beta_{1}(x) \chi_{1} \\
& \chi_{2}^{\prime}=\beta_{1}(x) \chi_{2}+\beta_{2}(x) \chi_{1},
\end{aligned}
$$

where $\beta_{1}(x)=f_{y}(x, \varphi(x))$ and $\beta_{2}(x)=f_{y y}(x, \varphi(x))$ are rational functions. This linear equation $(7)$ can be explicitly solved. Indeed, its solutions are given by

$$
\begin{aligned}
& \chi_{1}(x)=c_{1} \mathrm{e}^{2 \int \beta_{1}(x) d x}=c_{1} \omega^{2} \\
& \chi_{2}(x)=\mathrm{e}^{\int \beta_{1}(x) d x}\left(c_{1} \int \beta_{2}(x) \mathrm{e}^{\int \beta_{1}(x) d x} d x+c_{2}\right)=\omega\left(c_{1} \theta_{1}+c_{2}\right),
\end{aligned}
$$

\footnotetext{
${ }^{2}$ Since we are assuming that the variational equation has an irregular singular point at infinity, we have to compactify $\mathbb{C}^{2}$ and, consequently, this must be done in the projective complex plane.
} 
with $c_{1}, c_{2} \in \mathbb{C}$ arbitrary constants and provided we define

$$
\omega=\mathrm{e}^{\int \beta_{1}}, \quad \theta_{1}=\int \beta_{2} \omega .
$$

Assume now that equation (2) on the integral curve $\Gamma$ satisfy the following hypotheses:

(H1) Either the rational function $\beta_{1}(x)=R(x) / S(x)$ has a pole (at the finite complex plane) of order greater than 1 or $\operatorname{deg}(R) \leq \operatorname{deg}(S)$. Moreover, the residues of $\beta_{1}(x)$ at its poles must be all integers.

(H2) The function $\theta_{1}$ is not a rational function in the variables $x, \omega$.

We remark that the first part of hypothesis (H1) is equivalent to the fact that the variational equation $\mathrm{VE}_{1}, \chi_{1}^{\prime}=\beta_{1}(x) \chi_{1}$, has at least an irregular singularity in the extended complex plane, that is, a point in which vicinity the solution $\chi_{1}(x)$ displays an exponential behaviour of a rational function. The assumption about the residues is necessary in order that the integrand in (9), namely,

$$
\beta_{2}(x) \mathrm{e}^{\int \beta_{1}(x) d x},
$$

could be expressed as a product of a rational function by the exponential of a rational function. Although this assumption is not necessary for theoretical results, is very convenient and algorithmically relevant for the applications.

From this point onwards we will take, as the vector field of coefficients of the linear variational equations $\mathrm{LVE}_{k}$, the rational functions $\mathbb{C}(x)$. Remind that $G_{k}$ denotes the Galois group of $\mathrm{LVE}_{k}$, that is $G_{k}=\mathrm{Gal}\left(\mathrm{LVE}_{k}\right)$, and that $G_{k}^{0}$ is its corresponding identity component. Thus, the following result holds:

Proposition 3.1. Under assumptions (H1) and (H2) one has that $G_{2}=G_{2}^{0}$ and, moreover, $G_{2}$ is not abelian.

Proof. A fundamental matrix of system $\mathrm{LVE}_{2}$ is

$$
\Phi_{2}=\left(\begin{array}{cc}
\omega^{2} & 0 \\
\omega \theta_{1} & \omega
\end{array}\right)
$$

Consider $\sigma \in G_{2}$. Then by the action of $\sigma$ on the elements of the matrix $\Phi_{2}$ and by the definition of $G_{2}$ it must be $\sigma\left(\Phi_{2}\right)=\Phi_{2} B$ for some non singular complex matrix $B$. Now we are going to obtain the matrix $B$. As $\omega$ is a non trivial solution of the first order variational solution $\mathrm{LVE}_{1}$, it holds $\sigma(\omega)=\lambda \omega$, with $\lambda \in \mathbb{C}^{*}$ (see [4], Example 6.1.5) and $\sigma\left(\omega^{2}\right)=$ $(\sigma(\omega))^{2}=\lambda^{2} \omega^{2}$. Additionally,

$$
\left(\sigma\left(\theta_{1}\right)\right)^{\prime}=\sigma\left(\theta_{1}^{\prime}\right)=\sigma\left(\beta_{2} \omega\right)=\beta_{2} \sigma(\omega)=\lambda \beta_{2} \omega,
$$

and therefore $\sigma\left(\theta_{1}\right)=\lambda \theta_{1}+\mu$ for some $\mu \in \mathbb{C}$. So, we have, $\sigma\left(\omega \theta_{1}\right)=\sigma(\omega) \sigma\left(\theta_{1}\right)=\lambda \omega\left(\lambda \theta_{1}+\right.$ $\mu)=\lambda^{2} \omega \theta_{1}+\lambda \mu \omega$. Thus the Galois group $G_{2}$ is contained in the connected non abelian algebraic group

$$
M:=\left\{\left(\begin{array}{cc}
\lambda^{2} & 0 \\
\lambda \mu & \lambda
\end{array}\right): \lambda \in \mathbb{C}^{*}, \mu \in \mathbb{C}\right\}
$$


Recall that the dimension of the Galois group is equal to the transcendence degree of the Picard-Vessiot extension ( see [13], p. 394 or [4], Corollary 6.2.8). Since the Picard-Vessiot extension corresponding to $\mathrm{LVE}_{2}$ is

$$
\mathbb{C}(x) \subset \mathbb{C}(x, \omega)=L_{1} \subset \mathbb{C}\left(x, \omega, \theta_{1}\right)=L_{2},
$$

one has, from assumption (H1), that

$$
\operatorname{dim} G_{1}=\operatorname{tr} \operatorname{deg}\left(L_{1} / \mathbb{C}(x)\right)=\operatorname{tr} \operatorname{deg}(\mathbb{C}(x, \omega) / \mathbb{C}(x))=1 .
$$

So $G_{1} \simeq \mathbb{C}^{*}$. Now assumption (H2) implies that $\theta_{1} \notin \mathbb{C}(x, \omega)$, so the transcendence degree $\operatorname{tr} \operatorname{deg}\left(L_{2} / L_{1}\right)=1$. This means that the transcendence degree of the Picard-Vessiot extension $\mathbb{C}(x) \subset L_{2}$ is 2 and the dimension of the Galois group $G_{2}$ is also 2, but as $G_{2}$ is contained in $M$, they must coincide, that is $G_{2}^{0}=G_{2}=M$ is connected and not abelian.

Remark 3.2. In the proof of Proposition 3.1, $M$ is the semidirect product of the additive and the multiplicative groups, i.e., $M \simeq \mathbb{C}^{*} \ltimes \mathbb{C}$. Indeed, note that

$$
\left(\begin{array}{cc}
\lambda^{2} & 0 \\
\lambda \mu & \lambda
\end{array}\right)=\left(\begin{array}{cc}
\lambda^{2} & 0 \\
0 & \lambda
\end{array}\right) \cdot\left(\begin{array}{cc}
1 & 0 \\
\mu & 1
\end{array}\right)
$$

More information about semidirect products of algebraic groups can be found, for instance, in [9, Section 61].

Hence, assuming that one of the irregular singular points is at the infinity, by Theorem B we obtain the following corollary.

Corollary 3.3. Under the assumptions (H1) and (H2) it follows that the polynomial vector field $X$ (or its corresponding foliation) is not rationally integrable.

Now, using the Galoisian correspondence, it is possible to generalise Proposition 3.1 and Corollary 3.3 to higher order variational equations. Indeed, we denote by

$$
\beta_{k}(x)=f_{y \cdots y}(x, \varphi(x))(k \text {-times derivatives }), \quad \theta_{k-1}(x)=\int \beta_{k}(x) \omega^{k-1}(x) d x .
$$

Then, consider the following assumption (which generalises hypothesis (H2)):

(Hk) The function $\theta_{k-1}$ is not a rational function in the variables $x, \omega$.

Therefore, generalised versions of Proposition 3.1 and Corollary 3.3 become as follows:

Proposition 3.4. Under assumptions (H1) and (Hk) (for some $k \geq 2$ ) one has that $G_{k}=G_{k}^{0}$ and, moreover, $G_{k}$ is not abelian.

Theorem 3.5. Under the assumptions (H1) and (Hk) (for some $k \geq 2$ ) it follows that the polynomial vector field $X$ (or its corresponding foliation) is not rationally integrable. 
The proof of this theorem follows as a consequence of Proposition 3.4 and Theorem B.

Proof of Prop. 3.4. Instead of using the explicit linear representation of the Galois group $G_{k}$ it is more convenient to employ the Galoisian correspondence. So, the variational equation $\mathrm{VE}_{k}$ has the following structure

$$
\begin{aligned}
\varphi_{1}^{\prime} & =\beta_{1} \varphi_{1} \\
\varphi_{2}^{\prime} & =\beta_{1} \varphi_{2}+\beta_{2} \varphi_{1}^{2} \\
\varphi_{3}^{\prime} & =\beta_{1} \varphi_{3}+3 \beta_{2} \varphi_{1} \varphi_{2}+\beta_{3} \varphi_{1}^{3} \\
\vdots & \vdots \\
\varphi_{k}^{\prime} & =\beta_{1} \varphi_{k}+\cdots+\beta_{k} \varphi_{1}^{k}
\end{aligned}
$$

and its corresponding $\mathrm{LVE}_{k}$ becomes

$$
\begin{aligned}
\chi_{1}^{\prime} & =k \beta_{1} \chi_{1} \\
\vdots & \vdots \\
\chi_{k}^{\prime} & =\beta_{1} \chi_{k}+\cdots+\beta_{k} \chi_{1}
\end{aligned}
$$

with $\chi_{1}=\varphi_{1}^{k}, \ldots, \chi_{k}=\varphi_{k}$ and where only relevant terms have been explicitly written. From this structure and as it happens for $\mathrm{LVE}_{2}$, we see that the Picard-Vessiot extension $\mathbb{C}(x) \subset L_{k}$ of $\mathrm{LVE}_{k}$ is obtained by means of $\omega$ (exponential of a primitive) following some quadratures. Thus, the Picard-Vessiot extension of (13) is given by

$$
\mathbb{C}(x)=K \subset \mathbb{C}(x, \omega)=L_{1} \subset L_{2} \subset L_{3} \subset \cdots \subset L_{k},
$$

being $K \subset L_{1}$ given by adjoining $\omega$, the exponential of an integral. The rest, $L_{1} \subset L_{2}, \ldots$, $L_{k-1} \subset L_{k}$, by adjoining integrals (by means of variation of constants). Now assumption (H1) implies that the Galois group $G_{1}$ is (isomorphic to) the multiplicative group $\mathbb{C}^{*}$, and hence connected, $G_{1}=G_{1}^{0}=\mathbb{C}^{*}$ (see [4, Example 6.1.5]). The extension $L_{1} \subset L_{k}$ by integrals has a unipotent group $U$ as its Galois group (see [12]). Now by the Galois correspondence (we refer the reader to Appendix B), the group $G_{1}$ is isomorphic to the quotient $G_{k} / U$. As the groups $U$ and $G_{1}$ are connected, the group $G_{k}$ must be connected too.

Since $\chi_{1}=\omega^{k}$, and $\chi_{k}=c_{1} \omega+c_{2} \omega \theta_{k-1}+\cdots$, the solution of $\operatorname{LVE}_{k}$ must contain $\omega$ and $\theta_{k-1}$. Therefore, one has the intermediate extension

$$
\mathbb{C}(x) \subset \mathbb{C}\left(x, \omega, \theta_{k-1}\right) \subset L_{k} .
$$

We note that the extension $K=\mathbb{C}(x) \subset \mathbb{C}\left(x, \omega, \theta_{k-1}\right)=S$ is a Picard-Vessiot extension of the $2 \times 2$ linear system

$$
\begin{aligned}
& \bar{\chi}_{1}^{\prime}=k \beta_{1} \bar{\chi}_{1} \\
& \bar{\chi}_{k}^{\prime}=\beta_{1} \bar{\chi}_{k}+\beta_{k} \bar{\chi}_{1} .
\end{aligned}
$$

Let $H=\operatorname{Gal}\left(L_{k} / S\right)$ be the Galois group of the extension $S \subset L_{k}$ and $G=\operatorname{Gal}(S / K)$ the Galois group of equation (14). From hypotheses (H1) and (Hk) it is easy to prove that the group $G$ is not abelian (in fact, as for the second order variational equation, it is a semidirect product of the additive by the multiplicative group). So, again by the Galois correspondence of the Picard-Vessiot theory, $G \simeq G_{k} / H$. As $G$ is not abelian it follows that $G_{k}$ is not abelian as well. 
We point out that an alternative way to prove the connectedness of the group $G_{k}$ is by proving that $G_{k}$ is the semidirect product $G_{1} \ltimes U=\mathbb{C}^{*} \ltimes U$. But one needs then to use a more technical construction: the group $G_{k}$ can be considered as an algebraic subgroup of a jet group of local analytic diffeomorphisms over the complex plane (see [18]).

Remark 3.6. In Appendix $C$ the representation of the Galois group of the third variational equation is explicitly given. The reader can easily derive the proof of Proposition 3.4 for the case $k=3$.

Remark 3.7. It is worth to stress that in the proof of Proposition 3.4 it has not been used the second part of assumption (H1), i.e., that the residues of the primitive of $\beta_{1}$ must be integers. This assumption will become important later.

The results above draw a systematic scheme to check the non-rational integrability of a given polynomial vector field: first verifying (H1); then ckecking (H2) and if it is not satisfied go for (H3) and so on. If we find a $k \geq 2$ such that (Hk) holds, then our vector field is not rationally integrable.

As it will be seen later, equation (14) (which appears in a natural way in the previous proof) will play a crucial rôle. In fact, when facing a concrete family of polynomial systems, to check hypothesis (H1) will be quite straightforward by direct inspection of the rational function $\beta_{1}(x)$. However to check whether hypothesis (H2) (or, more generally, $(\mathrm{Hk})$ ) is satisfied is a much more involved difficulty. Next section is devoted to the relation between this problem and the existence of a rational solution of the so-called Risch equation.

\section{Liouville's TheOREM AND Risch Differential EQUATiON}

Let $f(x)$ and $g(x)$ be rational functions with $g(x)$ non constant. We say that the integral $\int f(x) \mathrm{e}^{g(x)} d x$ is elementary if it can be expressed in the form

$$
\int f(x) \mathrm{e}^{g(x)} d x=\mathrm{e}^{g(x)} h(x)+c,
$$

where $h(x)$ is a rational function and $c$ is an integration constant. The following assertion is a particular result from a more general theorem of Liouville.

Theorem $4.1([14])$. The integral $\int f(x) \mathrm{e}^{g(x)} d x$ is elementary if and only if the differential equation

$$
y^{\prime}+g^{\prime}(x) y=f(x)
$$

has a rational solution $h(x)$. More precisely, one has that $\int f(x) \mathrm{e}^{g(x)} d x=\mathrm{e}^{g(x)} h(x)+$ constant if and only if $h(x)$ satisfies equation (15).

Equation (15) is usually called the Risch differential equation, since he was the first one who provided an algorithm to decide whether it has or not a rational solution (see [20]). For a proof of this theorem using differential vector fields we refer the reader to [21, p.46]. In our setting we have $f(x)=\beta_{2}(x)$ and $g(x)=\int \beta_{1}(x) d x$. So, Risch equation (15) becomes

$$
y^{\prime}+\beta_{1}(x) y=\beta_{2}(x) .
$$


Hypothesis (H1) implies that $\omega$ can be reduced to a transcendental function of the form $h_{1}(x) \mathrm{e}^{g_{1}(x)}$, with $h_{1}$ and $g_{1}$ rational functions. Indeed, the function $h_{1}$ comes from the cancellation of the exponential with the possible logarithms defined by the poles of $\beta_{1}$ with integer residues. Thus, if this is the case, that is,

$$
\beta_{1}(x)=\tilde{\beta}_{1}(x)+\sum_{i} \frac{\ell_{i}}{x-x_{i}}
$$

with $\ell_{i} \in \mathbb{Z}$, then the primitive $\int \tilde{\beta}_{1}(x)$ is rational and

$$
\int \beta_{2}(x) \mathrm{e}^{\int \beta_{1}(x) d x}=\int \tilde{\beta}_{2}(x) \mathrm{e}^{\int \tilde{\beta}_{1}(x) d x} d x
$$

where $\tilde{\beta}_{2}(x)=\prod_{i}\left(x-x_{i}\right)^{\ell_{i}} \beta_{2}(x)$. Hence, by theorem 4.1, equation (16) should be changed by

$$
y^{\prime}+\tilde{\beta}_{1}(x) y=\tilde{\beta}_{2}(x) .
$$

For example, if we took $\beta_{1}(x)=3 /(x-1)+1 / x^{2}$ then $\mathrm{e}^{\int \beta_{1}(x) d x}=(x-1)^{3} \mathrm{e}^{\int 1 / x^{2} d x}$ and we would consider $\tilde{\beta}_{1}(x)=1 / x^{2}$ and would deal with the corresponding Risch equation $y^{\prime}+1 / x^{2} y=(x-1)^{3} \beta_{2}$.

On the other side, hypothesis (H2) is equivalent to say that the Risch equation (16) admits no rational solution. It is worth to mention that if (H1) applies and (H2) fails then there exists a unique rational solution $h(x)$ of the Risch equation. Indeed, the general solution of equation (15) is $y(x)=c \mathrm{e}^{-g(x)}+\mathrm{e}^{-g(x)} \int f(x) \mathrm{e}^{g(x)} d x, c \in \mathbb{C}$ a general constant. Since (H2) fails then $\int f(x) \mathrm{e}^{g(x)} d x$ is elementary and so $\int f(x) \mathrm{e}^{g(x)} d x=\mathrm{e}^{g(x)} h(x)$ and the general solution becomes $y(x)=c \mathrm{e}^{-\int \beta_{1}}+h(x)$, which is rational only for $c=0$.

Hence, by Theorem 4.1, assuming that hypothesis (H1) is satisfied, condition (H2) to be fulfilled is equivalent to the fact that the Risch equation (16) has no rational solution. The extension to $(\mathrm{Hk})$ is straightforward since its associated Risch equation is given by

$$
y^{\prime}+(k-1) \beta_{1}(x) y=\beta_{k}(x) .
$$

For algorithms dealing with this problem we refer the reader to the references $[6,22,11]$. We stress that there are usually two ways to approach this kind of issues:

a) Analytic approach, which tries to prove by analytic methods whether the equation (16) (or more general (17)) has or not any rational solution.

b) Algebraic approach, seeking to prove or disprove in a direct way the existence (or not) of a rational solution to equation (16) (or more general, for (17)). This is the way employed, essentially, in this work.

\section{VARIATIONAL EQUATIONS ALONG THE LINE AT INFINITY}

In this section we consider polynomial systems and study their variational equations along the line at infinity. We follow the ideas introduced in [8]. Let consider the polynomial vector field:

$$
\widetilde{X}=\widetilde{P}\left(z_{1}, z_{2}\right) \frac{\partial}{\partial z_{1}}+\widetilde{Q}\left(z_{1}, z_{2}\right) \frac{\partial}{\partial z_{2}}
$$


with

$$
\widetilde{P}\left(z_{1}, z_{2}\right)=\sum_{i=0}^{N_{1}} P_{i}\left(z_{1}, z_{2}\right), \quad \widetilde{Q}\left(z_{1}, z_{2}\right)=\sum_{i=0}^{N_{2}} Q_{i}\left(z_{1}, z_{2}\right),
$$

$P_{i}\left(z_{1}, z_{2}\right), Q_{i}\left(z_{1}, z_{2}\right)$ being homogeneous polynomials of degree $i$. Let define $N:=\max \left\{N_{1}, N_{2}\right\}$, the degree of $X$. The foliation defined by the vector field $\widetilde{X}$ is given by the first order differential equation

$$
\frac{d z_{2}}{d z_{1}}=\frac{\widetilde{Q}\left(z_{1}, z_{2}\right)}{\widetilde{P}\left(z_{1}, z_{2}\right)} .
$$

In case that $\widetilde{P} \equiv 0$ one should interchange the rôles of $z_{1}, z_{2}$ and $\widetilde{P}, \widetilde{Q}$. Two vector fields are said to be equivalent if they define the same foliation. Thus, in a dynamical language, we are more concerned with their orbits than with the time parametrisation of the integral curves of the vector field.

Proposition 5.1. For $z_{1} \neq 0$, the birational change of coordinates $y=1 / z_{1}$ and $x=y z_{2}=$ $z_{2} / z_{1}$ leads the polynomial vector field $\tilde{X}$ to the rational system

$$
\begin{aligned}
& \dot{x}=\sum_{i=0}^{N} y^{1-i}\left(Q_{i}(1, x)-x P_{i}(1, x)\right) \\
& \dot{y}=-\sum_{i=0}^{N} y^{2-i} P_{i}(1, x) .
\end{aligned}
$$

This change of variables sends the line at infinity $z_{1}=\infty$ to the line $y=0$. Its corresponding foliation is defined by the rational ordinary differential equation

$$
\frac{d y}{d x}=\frac{y \sum_{i=0}^{N} y^{N-i} P_{i}(1, x)}{\sum_{i=0}^{N} y^{N-i}\left(x P_{i}(1, x)-Q_{i}(1, x)\right)}
$$

and the associated polynomial vector field is given by

$$
X=P(x, y) \frac{\partial}{\partial x}+Q(x, y) \frac{\partial}{\partial y}
$$

where

$$
P(x, y):=\sum_{i=0}^{N} y^{N-i}\left(x P_{i}(1, x)-Q_{i}(1, x)\right), \quad Q(x, y):=y \sum_{i=0}^{N} y^{N-i} P_{i}(1, x) .
$$

Moreover, the following relations between $\widetilde{P}, \widetilde{Q}$ and $P, Q$ hold:

$$
P(x, y)=\frac{1}{z_{1}^{N+1}} \widetilde{P}\left(z_{1}, z_{2}\right), \quad Q(x, y)=\frac{1}{z_{1}^{N+1} z_{2}}\left(\widetilde{P}\left(z_{1}, z_{2}\right)+z_{1} \widetilde{Q}\left(z_{1}, z_{2}\right)\right),
$$

and

$$
\widetilde{P}\left(z_{1}, z_{2}\right)=\frac{1}{y^{N+1}} P(x, y), \quad \widetilde{Q}\left(z_{1}, z_{2}\right)=\frac{1}{y^{N+1}}(-y P(x, y)+x Q(x, y)) .
$$


Proof. From the change of variables it is clear that

$$
\begin{aligned}
\dot{x} & =\frac{1}{z_{1}} \dot{z}_{2}-\frac{z_{2}}{z_{1}} \frac{\dot{z}_{1}}{z_{1}}=\frac{1}{z_{1}}\left(\sum_{i=0}^{N} z_{1}^{i} Q_{i}\left(1, z_{2} / z_{1}\right)\right)-\frac{z_{2}}{z_{1}^{2}}\left(\sum_{i=0}^{N} z_{1}^{i} P_{i}\left(1, z_{2} / z_{1}\right)\right) \\
& =\sum_{i=0}^{N} y^{1-i}\left(Q_{i}(1, x)-x P_{i}(1, x)\right) .
\end{aligned}
$$

Analogously,

$$
\dot{y}=-\frac{\dot{z}_{1}}{z_{1}^{2}}=-y^{2} \sum_{i=0}^{N} y^{-i} P_{i}(1, x)=-\sum_{i=0}^{N} y^{2-i} P_{i}(1, x) .
$$

Notice that, however the polynomials $P\left(z_{1}, z_{2}\right)$ and $Q\left(z_{1}, z_{2}\right)$ were homogeneous in $z_{1}, z_{2}$ $P_{i}(1, x)$ and $Q_{i}(1, x)$, polynomials of degree at most $i$, are in general nonhomogeneous. The corresponding foliation of the system above is given by

$$
\frac{d y}{d x}=\frac{\dot{y}}{\dot{x}}=\frac{y \sum_{i=0}^{N} y^{N-i} P_{i}(1, x)}{\sum_{i=0}^{N} y^{N-i}\left(x P_{i}(1, x)-Q_{i}(1, x)\right)}=: \frac{Q(x, y)}{P(x, y)},
$$

where we have multiplied $\dot{y}$ and $\dot{x}$ by $y^{N-1}$. Concerning the relations between $\widetilde{P}, \widetilde{Q}$ and $P, Q$, one has

$$
\widetilde{P}\left(z_{1}, z_{2}\right)=\sum_{i=0}^{N} z_{1}^{i} P_{i}\left(1, z_{2} / z_{1}\right)=\frac{1}{y^{N+1}} P(x, y)
$$

or, equivalently,

$$
P(x, y)=\frac{1}{z_{1}^{N+1}} \widetilde{P}\left(z_{1}, z_{2}\right)
$$

In a similar way one gets

$$
\widetilde{Q}\left(z_{1}, z_{2}\right)=\sum_{i=0}^{N} z_{1}^{i} Q_{i}\left(1, z_{2} / z_{1}\right)=\frac{1}{y^{N+1}}(-y P(x, y)+x Q(x, y))
$$

and, taking into account the relation between $\widetilde{P}$ and $P$,

$$
Q(x, y)=\frac{1}{z_{1}^{N+1} z_{2}}\left(\widetilde{P}\left(z_{1}, z_{2}\right)+z_{1} \widetilde{Q}\left(z_{1}, z_{2}\right)\right) .
$$

So, in the end,

$$
\frac{d z_{2}}{d z_{1}}=\frac{\widetilde{Q}\left(z_{1}, z_{2}\right)}{\widetilde{P}\left(z_{1}, z_{2}\right)}=\frac{z_{2} Q\left(z_{2} / z_{1}, 1 / z_{1}\right)-P\left(z_{2} / z_{1}, 1 / z_{1}\right)}{z_{1} Q\left(z_{2} / z_{1}, 1 / z_{1}\right)}
$$


From now on, we restrict ourselves to polynomial vector fields $X$ of the form $(22)$ with $P(x, y)$, $Q(x, y)$ as in $(23)$ and having $y=0$ as an invariant curve. It is straightforward to check in this case that the corresponding second order linear variational equation $\mathrm{LVE}_{2}$ becomes

$$
\begin{aligned}
& \chi_{1}^{\prime}=2 \beta_{1}(x) \chi_{1} \\
& \chi_{2}^{\prime}=\beta_{1}(x) \chi_{2}+\beta_{2}(x) \chi_{1},
\end{aligned}
$$

with

$$
\begin{aligned}
& \beta_{1}(x)=\frac{P_{N}(1, x)}{x P_{N}(1, x)-Q_{N}(1, x)}, \\
& \beta_{2}(x)=2 \frac{P_{N}(1, x) Q_{N-1}(1, x)-P_{N-1}(1, x) Q_{N}(1, x)}{\left(x P_{N}(1, x)-Q_{N}(1, x)\right)^{2}} .
\end{aligned}
$$

\section{EXAMPLES AND ALGORITHMIC CONSIDERATIONS}

To illustrate the use of the method, we will focus our attention on those families of polynomial vector fields $X$ of the form

$$
\frac{d y}{d x}=\frac{y\left(P_{1}(x) y^{N-1}+\cdots+P_{N-1}(x) y+P_{N}(x)\right)}{x^{k}-y}
$$

or those families $\widetilde{X}$, as in (18), that can be led into this form by means of the birational change of variables $y=1 / z_{1}, x=z_{2} / z_{1}$. To avoid a cumbersome notation, hereafter we will also denote $P_{j}(x)=P_{j}(1, x)$.

Proposition 6.1. Let consider integers $N \geq 2,2 \leq k \leq N$ and a polynomial $\widetilde{P}$ of degree $N$,

$$
\widetilde{P}\left(z_{1}, z_{2}\right)=\sum_{i=0}^{N} P_{i}\left(z_{1}, z_{2}\right),
$$

with $P_{i}\left(z_{1}, z_{2}\right)$ homogeneous polynomial of degree $i$, satisfying that $P_{0}=0$ and $P_{i}\left(0, z_{2}\right)=0$, for $i=1, \ldots, N$. Let us define a polynomial of degree $N$,

$$
\widetilde{Q}\left(z_{1}, z_{2}\right)=\sum_{i=0}^{N} Q_{i}\left(z_{1}, z_{2}\right),
$$

where $Q_{i}\left(z_{1}, z_{2}\right)$, also homogeneous polynomials of degree $i$, are given by the following relations:

$$
\begin{aligned}
Q_{0}\left(z_{1}, z_{2}\right) & \equiv 0 \\
Q_{\ell}\left(z_{1}, z_{2}\right) & =\frac{z_{2}}{z_{1}} P_{\ell}\left(z_{1}, z_{2}\right), \quad \ell=1, \ldots, N-2 \\
Q_{N-1}\left(z_{1}, z_{2}\right) & =\frac{z_{2}}{z_{1}} P_{N-1}\left(z_{1}, z_{2}\right)+z_{1}^{N-1} \\
Q_{N}\left(z_{1}, z_{2}\right) & =\frac{z_{2}}{z_{1}} P_{N}\left(z_{1}, z_{2}\right)-z_{1}^{N-k} z_{2}^{k} .
\end{aligned}
$$


Under the transformation $y=1 / z_{1}, x=z_{2} / z_{1}$, the new polynomial vector field $X$ becomes

$$
X=\left(x^{k}-y\right) \frac{\partial}{\partial x}+y\left(P_{1}(x) y^{N-1}+\cdots+P_{N-1}(x) y+P_{N}(x)\right) \frac{\partial}{\partial y}
$$

and its foliation takes the form (27).

Proof. From Proposition 5.1 we know that under the transformation $y=1 / z_{1}, x=z_{2} / z_{1}$, any polynomial vector field $\widetilde{X}$ given by (18) and (19) takes the form

$$
\frac{d y}{d x}=\frac{y \sum_{i=0}^{N} y^{N-i} P_{i}(1, x)}{\sum_{i=0}^{N} y^{N-i}\left(x P_{i}(1, x)-Q_{i}(1, x)\right)} .
$$

So, in our case we should determine $\widetilde{Q}\left(z_{1}, z_{2}\right)$ such that

$$
\sum_{i=0}^{N} y^{N-i}\left(Q_{i}(1, x)-x P_{i}(1, x)\right)=x^{k}-y .
$$

Equating powers in $y$, one gets $Q_{0}=P_{0}=0$ and

$$
\begin{aligned}
Q_{\ell}(1, x) & =x P_{\ell}(1, x), \quad \ell=1, \ldots N-2, \\
Q_{N-1}(1, x) & =x P_{N-1}(1, x)+1, \quad Q_{N}(1, x)=x P_{N}(1, x)-x^{k} .
\end{aligned}
$$

Expressed in $\left(z_{1}, z_{2}\right)$-variables, they are led into the expression (28). Notice that the terms $\left(z_{2} / z_{1}\right) P_{j}\left(z_{1}, z_{2}\right)$ are well-defined polynomials since, by hypothesis, $P_{j}\left(0, z_{2}\right)=0$, for $j=$ $1, \ldots, N$.

From now on, we restrict ourselves to polynomial vector fields $X$ of degree $N$ whose foliation is given by an ordinary differential equation of the form (27),

$$
\frac{d y}{d x}=\frac{y\left(P_{1}(x) y^{N-1}+\cdots+P_{N-1}(x) y+P_{N}(x)\right)}{x^{k}-y}:=f(x, y)
$$

and with $2 \leq k \leq N$, an integer. As it was already pointed out at the end of Section 5 , its corresponding second order linear variational equation is given by (25). In our case the invariant curve is $\Gamma: y=0$, that is $y=\varphi(x)=0$, so

$$
\begin{aligned}
& \beta_{1}(x)=f_{y}(x, \varphi(x))=f_{y}(x, 0)=\frac{P_{N}(1, x)}{x^{k}} \\
& \beta_{2}(x)=f_{y y}(x, \varphi(x))=f_{y y}(x, 0)=2\left(\frac{P_{N}(1, x)}{x^{2 k}}+\frac{P_{N-1}(1, x)}{x^{k}}\right),
\end{aligned}
$$

as it was already stated in (26).

Example 1. Consider the planar vector field

$$
X=\left(x^{3}-y\right) \frac{\partial}{\partial x}+y\left(x^{2}-c x-b-a y\right) \frac{\partial}{\partial y}
$$


and its associated foliation defined by

$$
\frac{d y}{d x}=\frac{y\left(x^{2}-c x-b-a y\right)}{x^{3}-y} .
$$

Note that the vector field $X$ leaves invariant the straight line $y=0$. Now we consider the variational equations $\mathrm{LVE}_{2}$ of (31) on the line $y=0$ and we obtain

$$
\begin{aligned}
& \chi_{1}^{\prime}=2 \frac{x^{2}-c x-b}{x^{3}} \chi_{1} \\
& \chi_{2}^{\prime}=\frac{x^{2}-c x-b}{x^{3}} \chi_{2}-2\left(\frac{a}{x^{3}}-\frac{x^{2}-c x-b}{x^{6}}\right) \chi_{1} .
\end{aligned}
$$

Condition $b \neq 0$ implies hypothesis (H1). In order to check hypothesis (H2) we reduce our problem to study if its associated Risch equation

$$
y^{\prime}+\frac{x^{2}-c x-b}{x^{3}} y=-2\left(\frac{a}{x^{3}}-\frac{x^{2}-c x-b}{x^{6}}\right)
$$

has or not a rational solution.

Remark 6.2. For $b=0$ and $c \neq 0$, condition (H1) holds but (H2) fails. We have not find any $k$ that $\left(H_{k}\right)$ is verified.

Example 2 (an infinite family). Assume that in (27) $P_{N}=a \in \mathbb{C}$ and $P_{N-1}=b \in \mathbb{C}$. We assume $a \neq 0$ and $k \geq 2$. The vector field is

$$
X=\left(x^{k}-y\right) \frac{\partial}{\partial x}+y\left(P_{1}(x) y^{N-1}+\cdots P_{N-2}(x) y^{2}+b y+a\right) \frac{\partial}{\partial y}
$$

with foliation

$$
\frac{d y}{d x}=\frac{y\left(P_{1}(x) y^{N-1}+\cdots P_{N-2}(x) y^{2}+b y+a\right)}{x^{k}-y},
$$

$b \in \mathbb{C}, a \in \mathbb{C}^{*}$. Along the straight line $y=0$ its $\mathrm{LVE}_{2}$ becomes

$$
\begin{aligned}
\chi_{1}^{\prime} & =2 \frac{a}{x^{k}} \chi_{1} \\
\chi_{2}^{\prime} & =\frac{a}{x^{k}} \chi_{2}+2\left(\frac{a}{x^{2 k}}+\frac{b}{x^{k}}\right) \chi_{1} .
\end{aligned}
$$

As before, hypothesis (H2) and, therefore its rational integrability, can be reduced to study the existence of a rational solution of the corresponding Risch equation

$$
y^{\prime}+\frac{a}{x^{k}} y=\frac{2 a}{x^{2 k}}+\frac{2 b}{x^{k}} .
$$

We will prove later that this is not the case for $k>2$.

Example 3. Let $X$ be the following quadratic polynomial vector field:

Then

$$
X=y\left(a_{1} x+a_{0}\right) \frac{\partial}{\partial y}+\left(x^{2}-y\right) \frac{\partial}{\partial x} .
$$

$$
\frac{d y}{d x}=\frac{y\left(a_{1} x+a_{0}\right)}{x^{2}-y}
$$


Despite its simple appearance we will prove that generically the above vector field is not rationally integrable. Along the straight line $y=0$ the $\mathrm{LVE}_{2}$ becomes

$$
\begin{aligned}
\chi_{1}^{\prime} & =2 \frac{a_{1} x+a_{0}}{x^{2}} \chi_{1} \\
\chi_{2}^{\prime} & =\frac{a_{1} x+a_{0}}{x^{2}} \chi_{2}+\frac{2 a_{1} x+2 a_{0}}{x^{4}} \chi_{1} .
\end{aligned}
$$

As before, we study the existence of rational solutions for its corresponding Risch equation:

$$
y^{\prime}+\frac{a_{1} x+a_{0}}{x^{2}} y=2 \frac{a_{1} x+a_{0}}{x^{4}} .
$$

6.1. Application of the Risch-Kaltofen algorithm. In this section the solution of the Risch equation (16) is analysed by means of the so-called Risch algorithm. It follows the ideas of the work by Kaltofen in [11] (from now on refered as Risch-Kaltofen algorithm). We restrict ourselves here to the particular case where $\beta_{1}$ and $\beta_{2}$ are given by the expressions

$$
\begin{array}{ll}
\beta_{1}(x)=\frac{A(x)}{x^{k}}, & \beta_{2}(x)=\frac{2 A(x)+2 x^{k} B(x)}{x^{2 k}}, \\
A(x)=\sum_{i=0}^{n} a_{i} x^{i}, & B(x)=\sum_{i=0}^{m} b_{i} x^{i}, \quad k \in \mathbb{Z}^{+},
\end{array}
$$

with $a_{n} \neq 0, b_{m} \neq 0, k>1$ and $n<k$. From now on we will always consider $a_{0} \neq 0$ (if not, this would imply that $k$ becomes, at least, $k-1$ in $\left.\beta_{1}(x)\right)$. It is clear that $\beta_{1}(x)$ has a pole of order greater than 1 at $x_{0}=0$ (hypothesis (H1)).

Remark 6.3. Relevant special cases of the family (29) can be expressed in the latter form. For instance,

$$
\beta_{1}(x)=\frac{P_{N}(x)}{x^{k}}, \quad \beta_{2}(x)=2\left(\frac{P_{N}(x)}{x^{2 k}}+\frac{P_{N-1}(x)}{x^{k}}\right),
$$

with $n=\operatorname{deg} P_{N}(x), m=\operatorname{deg} P_{N-1}(x), n<k$. In particular, for $k=N+1$, we have $n=\operatorname{deg} P_{N}(x) \leq N<k$. However, as we will see later, there are polynomial systems not included in the family (29) with $\beta_{1}$ and $\beta_{2}$ as in (39).

Observe that $a_{0} \neq 0$ implies that $\operatorname{deg}\left(\operatorname{GCD}\left(A(x), x^{k}\right)\right)=0$ and also that $\operatorname{deg}(\operatorname{GCD}(2 A(x)+$ $\left.\left.2^{k} B(x), x^{2 k}\right)\right)=0$ (since they are irreducible fractions). In the same way, $b_{0} \neq 0$, unless $B(x)$ be identically zero, i.e., $B \equiv 0$. According to the notation of Kaltofen [11],

$$
q_{1}=x, k_{1}=k, l_{1}=2 k, F=A(x), G=2 A(x)+2 x^{k} B(x) .
$$

Due to $k>1$, then

$$
\tilde{j}_{1}=\min \{2 k+1, k\}=k, \quad y(x)=\frac{Y(x)}{x^{k}}
$$

and we arrive to the following differential equation $u Y^{\prime}+v Y=w$, i.e.,

$$
x^{k} Y^{\prime}+\left(A-k x^{k-1}\right) Y=2 A+2 x^{k} B,
$$

where

$$
\begin{array}{ll}
u=u_{p} x^{p}+\ldots+u_{0}=x^{k}, & v=v a_{r} x^{r}+\ldots+v_{0}=A-k x^{k-1} \\
w=w_{s} x^{s}+\ldots+w_{0}=2 A+2 x^{k} B, & Y=y_{h} x^{h}+\ldots+y_{0} .
\end{array}
$$


Replacing $x=0$ in equation (40) and having in mind that $a_{0} \neq 0$, it follows that $y_{0}=2$. Since $n<k, s<2 k$ it follows $m<k$ and $n \leq k-1$, and thus we arrive to

$$
p=k, \quad u_{p}=1, \quad r=k-1, \quad s= \begin{cases}m+k, & m \geq 0 \\ n, & B \equiv 0\end{cases}
$$

Let us define

$$
v_{r}= \begin{cases}-k, & n<k-1 \\ a_{n}-k, & n=k-1,\end{cases}
$$

and the function $\rho=-v_{r} / u_{p}$ as

$$
\rho=\left\{\begin{array}{cc}
-v_{r} & v_{r} \in \mathbb{Z}^{-} \\
0 & v_{r} \notin \mathbb{Z}^{-}
\end{array}\right.
$$

Therefore, equation (40) can be rewritten as

$$
\sum_{i=1}^{\widetilde{h}}\left[(i-k) x^{i+k-1}+A y_{i} x^{i}\right]=\sum_{i=0}^{m}\left(2 b_{i} x^{k+i}\right)+2 k x^{k-1},
$$

where $\widetilde{h} \geq h$ is given by

$$
\widetilde{h}= \begin{cases}\max \{\min \{m, m+1\}, \rho\} & \text { if } m \geq 0 \\ \max \{\min \{n-k-1, n-k+1\}, \rho\}=\max \{n-k-1, \rho\} & \text { if } B \equiv 0\end{cases}
$$

In our case, this $\widetilde{h}$ reads

$$
\widetilde{h}= \begin{cases}\max \{m, \rho\} & \text { if } m \geq 0, \\ \rho & \text { if } B \equiv 0 .\end{cases}
$$

One should obtain the rest of coefficients of $Y(x)$, that is $y_{\widetilde{h}}, y_{\widetilde{h}-1}, \ldots, y_{1}$, whenever the algebraic equation (40) has solution. Applying Rouché-Frobenius theorem over the system obtained after specialisation of the curve with $\widetilde{h}$ points $x_{1}, \ldots x_{\widetilde{h}}$ (where $x_{i} \neq 0$ for all $1 \leq i \leq$ $\widetilde{h}$ ) the polynomial $Y(x)$ exists if and only if the rank of the matrix of the system is exactly the rank of the augmented matrix. Recall that if

$$
\operatorname{deg}\left(x^{k} Y^{\prime}+\left(A-k x^{k-1}\right) Y\right) \neq \operatorname{deg}\left(2 A+2 x^{k} B\right),
$$

then there is no solution for $Y(x)$.

According to possibilities for $\widetilde{h}$ in equation (43) we have the following two cases:

(1) Case 1: $n<k-1$. This is the trivial case because $v_{r}=-k \in \mathbb{Z}^{-}$and since to $m<k$, it derives, by equation (43), that $\widetilde{h}=k$. By (42) we have

$$
\sum_{i=1}^{k}\left[(i-k) x^{i+k-1}+A x^{i}\right] y_{i}=\sum_{i=0}^{m}\left(2 b_{i} x^{k+i}\right)+2 k x^{k-1},
$$

which, after cancellation of the term of degree $2 k-1$, lead us to

$$
\begin{cases}-y_{k-1} x^{2 k-2}+\ldots+(1-k) y_{1} x^{k}+a_{n} y_{k} x^{n+k}+\ldots+a_{0} y_{1} x \equiv 0, & B \equiv 0 \\ -y_{k-1} x^{2 k-2}+\ldots+(1-k) y_{1} x^{k}+a_{n} y_{k} x^{n+k}+\ldots+a_{0} y_{1} x=2 b_{m} x^{m+k}+\ldots 2 b_{0} x^{k}, & m \geq 0\end{cases}
$$

Observe that for $B \equiv 0$, all the coefficients in the left-hand side must vanish in order to provide solution for $Y(x)$. On the other hand, for $m \geq 0$, the degree of the polynomial in the left-hand side is at most $2 k-2$ and the degree of the polynomial 
in the right-hand side is at most $m+k<2 k$. So, we can obtain rational solutions of Risch differential equation when equation (42) has solution for $Y(x)$. In particular, by condition (44), if the degree of the left-hand side is $2 k-2$, the degree of the righthand side is $m+k$ and since $m \neq k-2$ the Risch differential equation has no rational solution.

(2) Case 2: $n=k-1$

(a) If $m<1$ and $k-a_{n} \notin \mathbb{Z}^{+}$, then $\rho=0$ and by equation (43) we obtain $\widetilde{h}=0$. From (41) we get $Y(x)=y_{0}=2$. Replacing $Y(x)=2$ into equation (42) we obtain $x B=-k$, which is a contradiction because it does not exist $m \in \mathbb{N}$ such that $x B=-k$ (although $B \equiv 0$, because $k \neq 0$ ). Thus, we conclude that in this case there are no rational solutions for the Risch differential equation.

(b) If $k-a_{n} \notin \mathbb{Z}^{+}$and $m \geq 1$, then $\rho=0$ and by equation (43) we obtain $\widetilde{h}=m$. Using (41) we see that $Y(x)=y_{m} x^{m}+\ldots+2$ and replacing it into equation (42) we get rational solutions of Risch differential equation provided the rest of indeterminate coefficients $y_{1}, \ldots, y_{m}$ satisfy the algebraic equation (40).

(c) If $k-a_{n} \in \mathbb{Z}^{+}$and $m \geq k-a_{n}$, then $\rho=a_{n}-k$ and by equation (43) we obtain $\widetilde{h}=m$. Similarly, from (41) we see that $Y(x)=y_{m} x^{m}+\ldots+2$, and replacing it into equation (42), we obtain rational solutions of the Risch differential equation provided the rest of indeterminate coefficients $y_{1}, \ldots, y_{m}$ satisfy the algebraic equation (40).

(d) If $k-a_{n} \in \mathbb{Z}^{+}$and $m<k-a_{n}$, then $\rho=a_{n}-k$ and by equation (43) we obtain $\widetilde{h}=k-a_{n}$. Taking into account (41) one sees that $Y(x)=y_{k-a_{n}} x^{k-a_{n}}+\ldots+2$ and by replacing it into equation (42) one gets rational solutions for the Risch differential equation whenever the rest of indeterminate coefficients $y_{1}, \ldots, y_{k-a_{n}}$ satisfy the algebraic equation (40).

Now we go back to our families of examples.

Example 1 (continuation). Let us take $k=3, n=2, m=0, a_{2}=1, a_{1}=-c, a_{0}=-b$, $b_{0}=-a$ in equation (30). Therefore:

- In the case $a_{1} \neq a_{0} b_{0} / 3$ (equivalently, $c \neq-a b / 3$ ), it follows that $n=k-1$ and $m=0$ so we fall in case 2. Precisely, since $v_{r}=a_{2}-k=-2 \in \mathbb{Z}^{-}$, we fall in subcase 2.d, which lead us to $\widetilde{h}=2$. Hence, $Y(x)=y_{2} x^{2}+y_{1} x+2$. Now, substituting $Y(x)$ into equation (42) we see that condition (44) holds and Risch differential equation has no rational solution. In consequence, equation (30) is not rationally integrable. Coming back to the vector field $\widetilde{X}\left(z_{1}, z_{2}\right)$, by means of equation (24), we obtain that the vector field

$$
\widetilde{X}=\left(a b z_{1}^{2}+c z_{1} z_{2}-z_{2}^{2}+z_{1}\right) \frac{\partial}{\partial z_{1}}-\left(c z_{2}^{2}+a b z_{1} z_{2}+z_{2}-z_{1}\right) \frac{\partial}{\partial z_{2}}
$$

is not rationally integrable. In fact, the vector field (45) is one of the equivalent vector fields defining the same foliation.

- In the case $a_{1}=a_{0} b_{0} / 3$ (equivalently, $c=-a b / 3$ ), the rational solution of the Risch equation is given by $y(x)=\frac{-\frac{6}{b} x^{2}+2}{x^{3}}$. Thus, hypothesis (H2) is not fulfilled and the method does not decide. 
Example 2 (continuation). Take $n=m=0, A=a$ and $B=b$ in equation (34). Due to $n<k-1$, we fall in case 1 . Thus, $\widetilde{h}=k$ and $Y(x)=y_{k} x^{k}+\ldots+y_{1} x+2$. If condition (44) is satisfied, then $2 k-2 \neq k$, which implies that we have not rational solution for all $k>2$.

Now, since $k=2$ does not satisfy condition (44), we seek for $Y(x)$ using equations (40) or (42). We see that $\widetilde{h}=2$ and $Y(x)=y_{2} x^{2}+y_{1} x+2$. Now, by equation (42), we obtain

$$
y_{1}=\frac{4}{a}, \quad y_{2}=\frac{2 a b+4}{a^{2}} .
$$

The solution is given by

$$
y(x)=\frac{2}{x^{2}}+\frac{4}{a x}+\frac{2 b}{a}+\frac{4}{a^{2}}+\mathrm{e}^{\frac{a}{x}} c_{1} .
$$

Thus, for this infinite family, the Risch equation has a rational solution for $k=2$ and no rational solution for $k>2$. Therefore, it follows that the vector field

$$
\widetilde{X}=\widetilde{P}\left(z_{1}, z_{2}\right) \frac{\partial}{\partial z_{1}}+\widetilde{Q}\left(z_{1}, z_{2}\right) \frac{\partial}{\partial z_{2}},
$$

with

$$
\widetilde{P}\left(z_{1}, z_{2}\right)=\sum_{i=0}^{N_{1}} P_{i}\left(z_{1}, z_{2}\right), \quad \widetilde{Q}\left(z_{1}, z_{2}\right)=\sum_{i=0}^{N_{2}} Q_{i}\left(z_{1}, z_{2}\right),
$$

where $P_{i}\left(z_{1}, z_{2}\right), Q_{i}\left(z_{1}, z_{2}\right)$ are homogeneous polynomials of degree $i$, and satisfying conditions (28) is not rationally integrable if $P_{N}\left(z_{1}, z_{2}\right)=a z_{1}^{N}$ and $P_{N-1}\left(z_{1}, z_{2}\right)=b z_{1}^{N-1} z_{2}-z_{1}^{N-1}$.

Example 3 (continuation). Take $k=2, A=a_{1} x+a_{0}$ (with $\left.a_{1} \neq 0\right), B \equiv 0, n=1$, and

$$
\beta_{1}=\frac{a_{1} x+a_{0}}{x^{2}}, \quad \beta_{2}=\frac{2 a_{1} x+2 a_{0}}{x^{4}},
$$

in equation (38). Since $n=k-1$ (case 2 of the algorithm) and $m<1$ we should consider two options. The first one is when $a_{1}-2$ is not a negative integer, that is $a_{1} \in[2, \infty)$, we fall in subcase 2.a and therefore the Risch differential equation has no rational solutions.

Now, the second option is when $a_{1}-2$ is a negative integer, that is $a_{1} \in(-\infty, 1] \cap \mathbb{Z}$ and we fall in subcase 2.d. Thus, $\widetilde{h}=2-1>0$ and then the Risch differential equation has always one rational solution because $y_{m}, \ldots, y_{1}$ satisfy the algebraic equation (42) and we can obtain explicitly the polynomial $Y(x)$, which lead us to the rational solution $y(x)=\frac{Y(x)}{x^{2}}$. For instance, setting $a_{1}=1$ and $a_{0}=a$, we obtain $\widetilde{h}=1$ and $Y(x)=\frac{4}{a} x+2$ and the rational solution of the Risch differential equation is

$$
y(x)=\frac{4}{a x}+\frac{2}{x^{2}} .
$$

Coming back to the variables $\left(z_{1}, z_{2}\right)=(1 / y, x / y)$ the vector field (37) is (equivalent to)

$$
\widetilde{X}=\left(a_{0} z_{1}^{2}+a_{1} z_{1} z_{2}\right) \frac{\partial}{\partial z_{1}}-\left(a_{1} z_{2}^{2}+a_{0} z_{1} z_{2}-z_{2}^{2}+z_{1}\right) \frac{\partial}{\partial z_{2}} .
$$

Thus we proved that for $a_{1} \notin(-\infty, 1] \cap \mathbb{Z}$ the vector field (46) is not rationally integrable. We observe that for $a_{1}=1$ it reduces to a vector field equivalent to the following linear one

$$
\widetilde{X}=\left(a_{0} z_{1}+z_{2}\right) \frac{\partial}{\partial z_{1}}-\left(a_{0} z_{2}+1\right) \frac{\partial}{\partial z_{2}} .
$$




\section{Conjecture: Extensions to other type of First integrals}

We believe that our results provide obstructions for a more general kind of first integrals. More precisely, it seems natural to think assumptions (H1) and (mainly) (Hk) ( $k \geq 2$ ) are not compatible with the existence of an elementary first integral (for a precise definition of it, see for instance [19]). Indeed, the integral $\theta_{k}$ is too "transcendent" to allow an elementary first integral. Then we state the following:

Conjecture. Under assumptions (H1) and (Hk) (for some $k \geq 2$ ) the polynomial vector field has not an elementary first integral.

We illustrate this conjecture with the vector field (37) with $a_{1}=1$. Since equation (47) is linear in the variables $z_{1}, z_{2}$, it must be integrable in some reasonable sense.

Example. The function

$$
H(x, y)=\frac{a x+y}{y} \mathrm{e}^{-\frac{a(x+a)}{a x+y}}
$$

is a first integral of the vector field

$$
X=\left(x^{2}-y\right) \frac{\partial}{\partial x}+y(x+a) \frac{\partial}{\partial y} .
$$

Notice that $H$ is elementary, in fact of Darboux type (see $[5,7]$ ).

From the previous section we know that assumption (H1) is satisfied but not (H2), i.e, $\theta_{1}$ is elementary. We compute $\theta_{k-1}$ up to $k=10$ and all them are elementary (we skip the details), i.e, no assumption ( $\mathrm{Hk}$ ) is satisfied for $k \leq 10$ and hence until $k=10$ no obstruction to rational integrability is obtained, despite of the fact that the first integral is not rational but elementary.

\section{Appendix A: Some Maple computations}

We will give some flavour of the analytical approach using MAPLE on two of the examples of Section 6: Example 1 and Example 3. The results are in agreement with the algebraic results of Section 6.1.

Example 1. There are two possible cases: (i) $c \neq-a b / 3$ and (ii) $c=-a b / 3$.

(i) If $b \neq 0$ and $c \neq-a b / 3$ the solution of $\mathrm{LVE}_{2}$ is

$$
\begin{aligned}
& \chi_{1}(x)=c_{1} e^{\frac{2 c x+b}{x^{2}}} x^{2}, \\
& \chi_{2}(x)=c_{2} e^{\frac{2 c x+b}{2 x^{2}}} x+c_{1} \sqrt{2 \pi} \frac{\operatorname{erf}\left(\frac{\sqrt{2}(c x+b)}{2 \sqrt{-b x}}\right)(a b+3 c) e^{\frac{-c^{2} x^{2}+2 b c x+b^{2}}{2 b x^{2}}} x+\sqrt{2} \sqrt{-b} e^{\frac{2 c x+b}{x^{2}}}\left(3 x^{2}-b\right)}{(-b)^{3 / 2} x},
\end{aligned}
$$

and notice that for $b \neq 0$ the error function $\operatorname{erf}\left(\frac{\sqrt{2}(c x+b)}{2 x \sqrt{-b}}\right)$ is not an elementary function (see, for instance, [21, page 48]). We remark that it is also possible to express the error function by means of the Whittaker function ([26]) $W_{-1 / 4,1 / 4}$ (and some elementary functions). Then by proving that this Whittaker function is not elementary, we obtain an alternative proof of the non-elementary character of the error function. Notice that $G_{2}=\operatorname{Gal}\left(\mathrm{LVE}_{2}\right)$ is connected and non abelian. Therefore, the vector field $X$ is no rationally integrable. 
(ii) In the case $c=-a b / 3$, the solution of $\mathrm{LVE}_{2}$ is

$$
\begin{aligned}
& \chi_{1}(x)=c_{1} x^{2} \mathrm{e}^{-\frac{b(2 a x-3)}{3 x^{2}}}, \\
& \chi_{2}(x)=\frac{1}{b x}\left(2 c_{1} \mathrm{e}^{-\frac{b(2 a x-3)}{6 x^{2}}}\left(-3 x^{2}+b\right)+c_{2} b x^{2}\right) \mathrm{e}^{-\frac{b(2 a x-3)}{6 x^{2}}} .
\end{aligned}
$$

Solutions are elementary and its associated Risch equation has a rational solution.

Example 3. In this family the $\mathrm{LVE}_{2}$ becomes

$$
\begin{aligned}
\chi_{1}^{\prime} & =2 \frac{a_{1} x+a_{0}}{x^{2}} \chi_{1} \\
\chi_{2}^{\prime} & =\frac{a_{1} x+a_{0}}{x^{2}} \chi_{2}+2 \frac{a_{1} x+a_{0}}{x^{4}} \chi_{1} .
\end{aligned}
$$

MAPLE gives the following solution of $\mathrm{LVE}_{2}$

$$
\begin{aligned}
& \chi_{1}(x)=c_{1} x^{2 a_{1}} e^{-2 \frac{a_{0}}{x}} \\
& \chi_{2}(x)=c_{2} x^{a_{1}} e^{-\frac{a_{0}}{x}}+c_{1} \frac{2 a_{0}^{2} e^{\frac{-2 a_{0}}{x}} x^{2 a_{1}-2}+4 a_{0} e^{\frac{-2 a_{0}}{x}} x^{2 a_{1}-1}-4\left(\frac{a_{0}}{x}\right)^{\frac{a_{1}}{2}} M e^{-\frac{3 a_{0}}{2 x}} x^{2 a_{1}}}{a_{0}^{2}},
\end{aligned}
$$

where we have denoted by

$$
M:=M_{\kappa, \mu}(z), \quad \text { with } \quad \kappa=-\frac{a_{1}}{2}, \mu=\frac{1-a_{1}}{2}, z=\frac{a_{0}}{x},
$$

the M Whittaker function solution of the Whittaker equation

$$
y^{\prime \prime}-\left(\frac{1}{4}-\frac{\kappa}{z}+\frac{4 \mu^{2}-1}{4 z^{2}}\right) y=0 .
$$

In all the checked values for $a_{1} \in[-\infty, 1] \cap Z$ the $\mathrm{M}$ function is elementary and it is not elementary for the rest of values, in agreement with our previous results.

\section{Appendix B: The Galoisian Correspondence}

One of the key theorems of the Galois Theory of linear differential equations, the PicardVessiot Theory, is the existence of a Galoisian correspondence between intermediate differential field in the Picard-Vessiot extension of a linear differential system and algebraic subgroups of the Galois group. It means that the structure of the solutions of the differential equation (i.e., how to solve the differential equation) is a reflection of the structure of Galois group. An analogous theorem is valid for the classical Galois Theory of polynomials and as there, this theorem plays an essential rôle not only in the theory but also in applications. For this reason this theorem is also called the Fundamental Theorem of Picard-Vessiot Theory (some references are $[15,4,24])$.

We need some terminology and notations. Let $K \subset L$ be an extension of differential fields. Then:

- $\operatorname{Gal}(L / K)$ is the group of differential automorphisms of $L$ which are the identity over $K$.

- If $H$ is a subgroup of $\operatorname{Gal}(L / K)$, the intermediate field $K \subset L^{H} \subset L$ fixed by $H$ is $L^{H}:=\{a \in L: \sigma(a)=a, \forall \sigma \in H\}$. 
- Given a linear differential system

$$
\frac{d \mathbf{y}}{d x}=A \mathbf{y}
$$

with coefficients in a differential field $K$ (that is $A \in \mathrm{Mat}(n, K)$ ), a Picard-Vessiot extension $K \subset L$ of equation (48) is the extension generated by the elements of a fundamental matrix $\Phi(x)=\left(\phi_{i j}(x)\right), L=K\left(\phi_{11}, \ldots, \phi_{n n}\right)$. From equation (48), the field $L$ is a differential field.

Theorem (Correspondence in Picard-Vessiot theory)

Let $K \subset L$ be a Picard-Vessiot extension of (48) and $G=G a l(L / K)$. Then there exists a one-to-one correspondence between the intermediary differential fields $K \subset S \subset L$ and the algebraic subgroups $H \subset G$ such that:

1) Given $S, H=\operatorname{Gal}(L / S)$.

2) Given $H, L^{H}=S$.

3) Picard-Vessiot extensions $K \subset S$ correspond to normal subgroups $H \subset G$ and then $G / H \approx \operatorname{Gal}(S / K)$.

4) If $M$ is a subgroup of $G$, then to the differential field $L^{M}$ it corresponds the Zariski adherence of $H$, i.e., $\operatorname{Gal}\left(L / L^{M}\right)=\bar{H}$.

\section{APPEndix C: THE GALOIS GRUP of THE THIRD ORDER VARIATIONAL EQUATION}

Linearising the variational equations. Variational equations and their linearised expression play a key rôle in the theory of integrability of differential equations and, in particular, they have been also crucial in the results of this work. However they are very well known and commonly used in many textbooks on differential equations, higher order variational equations and (a possible, there can be several) their linearisation are not so frequent. The aim of this annex is to remind them in order to do this paper a bit more self-contained. We illustrate the method with the third order variational equation.

To start, let us assume that $y=\varphi(x)$ is a solution of $y^{\prime}=f(x, y), y\left(x_{0}\right)=\varphi\left(x_{0}\right)=y_{0}$. One possible way to derive the variational equations related to the $y$-variable (that is, freezing $x_{0}$ as a initial $x$-value and moving transversally in the $y$-direction, that is, $y\left(x_{0}\right)=y_{0}+\varepsilon$ ) is to look for solutions of such equation in the perturbative form, more suitable for computations that the way we deduced the $\mathrm{VE}_{2}$ in the introduction,

$$
y(x)=\varphi(x)+\varepsilon \varphi_{1}(x)+\frac{\varepsilon^{2}}{2 !} \varphi_{2}(x)+\cdots+\frac{\varepsilon^{j}}{j !} \varphi_{j}(x)+\cdots
$$


Substituting it into $y^{\prime}=f(x, y)$ and expanding in Taylor series we get

$$
\begin{gathered}
\varphi^{\prime}(x)+\varepsilon \varphi_{1}^{\prime}(x)+\frac{\varepsilon^{2}}{2 !} \varphi_{2}^{\prime}(x)+\frac{\varepsilon^{3}}{3 !} \varphi_{3}^{\prime}(x)+\cdots= \\
f(x, \varphi)+f_{y}(x, \varphi)\left(\varepsilon \varphi_{1}+\frac{\varepsilon^{2}}{2 !} \varphi_{2}+\frac{\varepsilon^{3}}{3 !} \varphi_{3}+\cdots\right)+ \\
\frac{1}{2 !} f_{y y}(x, \varphi)\left(\varepsilon \varphi_{1}+\frac{\varepsilon^{2}}{2 !} \varphi_{2}+\frac{\varepsilon^{3}}{3 !} \varphi_{3}+\cdots\right)^{2}+ \\
\frac{1}{3 !} f_{y y y}(x, \varphi)\left(\varepsilon \varphi_{1}+\frac{\varepsilon^{2}}{2 !} \varphi_{2}+\frac{\varepsilon^{3}}{3 !} \varphi_{3}+\cdots\right)^{3}+\cdots
\end{gathered}
$$

where $f_{y}(x, y)=\partial f / \partial y, f_{y y}=\partial^{2} f / \partial y^{2}$, etc. Equating terms of power $\varepsilon^{k}$ one obtains the variational equations of any order $k \geq 1$. Indeed, for $k=0$ one get the known solution $\varphi^{\prime}(x)=f(x, \varphi(x))$. Concernng the rest of orders one has:

$$
\begin{aligned}
& \varphi_{1}^{\prime}=f_{y}(x, \varphi) \varphi_{1} \\
& \varphi_{1}\left(x_{0}\right)=1 \\
& \varphi_{2}^{\prime}=f_{y}(x, \varphi) \varphi_{2}+f_{y y}(x, \varphi) \varphi_{1}^{2} \\
& \varphi_{2}\left(x_{0}\right)=0, \\
& \varphi_{3}^{\prime}=f_{y}(x, \varphi) \varphi_{3}+3 f_{y y}(x, \varphi) \varphi_{1} \varphi_{2}+f_{y y y}(x, \varphi) \varphi_{1}^{3} \\
& \varphi_{3}\left(x_{0}\right)=0,
\end{aligned}
$$

and so on. The initial values come from the condition $y\left(x_{0}\right)=y_{0}+\varepsilon$. Observe that its solution exhibits a triangular scheme: we substitute the functions $\varphi_{j}, j=1, \ldots, k-1$ previously obtained in the equation for $\varphi_{k}$ and solve for it. Thus, all of them are non-homogeneous linear differential equations except the first one, $\mathrm{VE}_{1}$, which is homogeneous. They can be solved recurrently using the exponential of a primitive (for the first variational equation) and primitives.

Nevertheless they can be computed (specially, numerically) in this form, their expression is not the most suitable when regarding them in terms of differential Galois Theory. In that framework, the common approach is to introduce intermediate variables which lead equations $\mathrm{VE}_{k}, k \geq 2$, into linear homogeneous systems. Namely, for the $\mathrm{VE}_{3}$ let us define the following variables

$$
\chi_{1}=\varphi_{1}^{3}, \quad \chi_{2}=\varphi_{1} \varphi_{2}, \quad \chi_{3}=\varphi_{3} .
$$

Thus, the third order variational equation $\mathrm{VE}_{3}$ becomes the $\mathrm{LVE}_{3}$

$$
\begin{aligned}
\chi_{1}^{\prime} & =3 \varphi_{1}^{2} \varphi_{1}^{\prime}=3 f_{y}(x, \varphi) \varphi_{1}^{3}=3 f_{y}(x, \varphi) \chi_{1} \\
\chi_{2}^{\prime} & =\varphi_{1}^{\prime} \varphi_{2}+\varphi_{1} \varphi_{2}^{\prime}=f_{y}(x, \varphi) \varphi_{1} \varphi_{2}+\varphi_{1}\left(f_{y}(x, \varphi) \varphi_{2}+f_{y y}(x, \varphi) \varphi_{1}^{2}\right) \\
& =2 f_{y}(x, \varphi) \chi_{2}+f_{y y}(x, \varphi) \chi_{1} \\
\chi_{3}^{\prime} & =f_{y}(x, \varphi) \chi_{3}+3 f_{y y}(x, \varphi) \chi_{2}+f_{y y y}(x, \varphi) \chi_{1},
\end{aligned}
$$


which gives rise to the following triangular system

$$
\left(\begin{array}{c}
\chi_{1}^{\prime} \\
\chi_{2}^{\prime} \\
\chi_{3}^{\prime}
\end{array}\right)=\left(\begin{array}{ccc}
3 f_{y}(x, \varphi) & 0 & 0 \\
f_{y y}(x, \varphi) & 2 f_{y}(x, \varphi) & 0 \\
f_{y y y}(x, \varphi) & 3 f_{y y}(x, \varphi) & f_{y}(x, \varphi)
\end{array}\right)\left(\begin{array}{c}
\chi_{1} \\
\chi_{2} \\
\chi_{3}
\end{array}\right) .
$$

For variational equations of higher order a similar procedure can be performed.

Galois group of the third order linearised variational equation $\mathbf{L V E}_{3}$. We recall that

$$
\beta_{1}(x)=f_{y}(x, \varphi(x)), \quad \beta_{2}(x)=f_{y y}(x, \varphi(x)), \quad \beta_{3}(x)=f_{y y y}(x, \varphi(x)),
$$

and

$$
\omega=\mathrm{e}^{\int \beta_{1} d x}, \quad \theta_{1}=\int \beta_{2} \mathrm{e}^{\int \beta_{1} d x} d x, \quad \theta_{2}=\int \beta_{3} \mathrm{e}^{2 \int \beta_{1} d x} d x
$$

Observe that

$$
\omega=\mathrm{e}^{\int \beta_{1} d x}, \quad \theta_{1}=\int \beta_{2} \omega, \quad \theta_{2}=\int \beta_{3} \omega^{2} .
$$

To solve the linear differential equations $\mathrm{LVE}_{3}$ (homogeneous the first one and nonhomogeneous the others) we use that the general solution of a linear ordinary differential equation $y^{\prime}=$ $a(x) y+b(x)$ is given, using the formula of variation of parameters, by $y=c \mathrm{e}^{\int a}+\mathrm{e}^{\int a} \int b \mathrm{e}^{-\int a}$. Indeed, we have

$$
\begin{aligned}
& \chi_{1}=c_{1} \omega^{3}, \\
& \chi_{2}=c_{2} \omega^{2}+c_{1} \omega^{2} \theta_{1}, \\
& \chi_{3}=c_{3} \omega+3 c_{2} \omega \theta_{1}+c_{1} \omega \theta_{2}+\frac{3}{2} c_{1} \omega \theta_{1}^{2},
\end{aligned}
$$

where in the last equation it has been used that

$$
\int \beta_{2} \omega \theta_{1}=\int \beta_{2} \mathrm{e}^{\int \beta_{1}} \int \beta_{2} \mathrm{e}^{\int \beta_{1}}=\int \theta_{1} \theta_{1}^{\prime}=\frac{1}{2} \theta_{1}^{2}=\frac{1}{2}\left(\int \beta_{2} \mathrm{e}^{\int \beta_{1}}\right)^{2}
$$

and with $c_{1}, c_{2}, c_{3}$ complex constants. That is, a possible fundamental matrix of system $\mathrm{LVE}_{3}$ is given by

$$
\Phi_{3}=\left(\begin{array}{ccc}
\omega^{3} & 0 & 0 \\
\omega^{2} \theta_{1} & \omega^{2} & 0 \\
\frac{3}{2} \omega \theta_{1}^{2}+\omega \theta_{2} & 3 \omega \theta_{1} & \omega
\end{array}\right)
$$

In what follows we work by a similar way to the case of second order variational equation, see Proposition 3.1. Consider $\sigma \in G_{3}$ (the Galois group of $\mathrm{LVE}_{3}$ ). It must be $\sigma\left(\Phi_{3}\right)=\Phi_{3} B$, being $B$ a non singular complex matrix. Following similar arguments to the computation of $G_{2}$ in the proof of Proposition 3.1 we obtain $\sigma(\omega)=\lambda \omega, \sigma\left(\theta_{1}\right)=\lambda \theta_{1}+\mu, \sigma\left(\theta_{2}\right)=\lambda^{2} \theta_{2}+\nu$, with $\lambda \neq 0$ and $\mu, \nu$ suitable complex constants. It follows that the corresponding Galois group $G_{3}$ is represented by an algebraic subgroup of the non abelian connected algebraic group

$$
M=\left\{\left(\begin{array}{ccc}
\lambda^{3} & 0 & 0 \\
\lambda^{2} \mu & \lambda^{2} & 0 \\
\lambda \nu+\frac{3}{2} \lambda \mu^{2} & 3 \lambda \mu & \lambda
\end{array}\right): \lambda \in \mathbb{C}^{*}, \mu \in \mathbb{C}, \nu \in \mathbb{C}\right\} .
$$


Now assumption (H1) implies that the dimension of $G_{1}$ is 1 (see again the proof of Proposition 3.1). Since the dimension of the group $G_{3}$ must be equal to the transcendence degree of the extension $\mathbb{C}(x) \subset L_{3}$, it is easy to see that assumptions (H2), (H3) are not verified if and only if $\mu=0$ and $\nu=0$, respectively. Then the structure of the Galois group $G_{3}$ will be as follows

$$
G_{3}=\left\{\left(\begin{array}{ccc}
\lambda^{3} & 0 & 0 \\
0 & \lambda^{2} & 0 \\
\lambda \nu & 0 & \lambda
\end{array}\right): \lambda \in \mathbb{C}^{*}, \nu \in \mathbb{C}\right\},
$$

assuming (H3) but not (H2), and

$$
G_{3}=\left\{\left(\begin{array}{ccc}
\lambda^{3} & 0 & 0 \\
\lambda^{2} \mu & \lambda^{2} & 0 \\
\frac{3}{2} \lambda \mu^{2} & 3 \lambda \mu & \lambda
\end{array}\right): \lambda \in \mathbb{C}^{*}, \mu \in \mathbb{C}\right\},
$$

assuming (H2) but not (H3). In any of these two cases, the group $G_{3}$ is connected and not abelian. For the applications, when (H1) is satisfied and (H2) is not satisfied then we check (H3). If (H3) is satisfied and since the group $G_{3}^{0}=G_{3}$ in (51) is not abelian, we conclude to the non rational integrability.

\section{ACKNOWLEDGEMENTS}

JM is member of the Universidad Politécnica de Madrid research group "Modelos Matemáticos no lineales". His work has been partially supported by this research group. CP has been partially supported by Spanish MECC-FEDER grant MTM2015-65715-P and MINECO-FEDER grant MTM2016-77278-P, and by the Catalan AGAUR grant 2014SGR-504. JTL has been supported by Spanish MECC-FEDER grant MTM2015-65715-P, the Catalan AGAUR grant 2014SGR-504 and the grant 14-41-00044 of RSF at the Lobachevsky University of Nizhny Novgorod (Russia). PB thanks Universidad Simón Bolívar (Barranquilla, Colombia) for its support during the final stage of this work. All the authors thank the EPSEB (UPC) for its support.

And last, but not least, the authors would like to thank the people from the UPM Integrability Seminar in Madrid, the UB-UPC Dynamical Systems Seminar in Barcelona and the Dynamical Systems Seminar at the UAB in Bellaterra, where preliminary versions of this work were exposed. In particular to C. Simó and A. Delshams for their interesting remarks and suggestions.

\section{REFERENCES}

[1] P. B. Acosta-Humánez, J.T. Lázaro, J. Morales-Ruiz and Ch. Pantazi, On the integrability of polynomial vector fields in the plane by means of Picard-Vessiot theory, Discrete and Continuous Dynamical Systems - Series A (DCDS-A), 35 (2015),No. 5, 1767-1800. DOI: 10.3934/dcds.2015.35.1767

[2] M. Ayoul, N.T. Zung, Galoisian obstructions to non-Hamiltonian integrability, C. R. Math. Acad. Sci. Paris, 348 (2010) 1323-1326.

[3] G. Casale, Morales-Ramis Theorems via Malgrange pseudogroup, Ann. Inst. Fourier,Tome 59, 7 (2009)2593-2610. DOI: 10.1016/j.crma.2010.10.024

[4] C. Crespo, Z. Hajto, Algebraic Groups and Differential Galois Theory, American Mathematical Society, Rhode Island, 2011.

[5] G. Darboux, Mémoire sur les équations différentielles algébriques du premier ordre et du premier degré (Mélanges), Bull. Sci. math. 2ème série, 2 (1878) 60-96; 123-144; 151-200. 
[6] J.H. Davenport, The Risch Differential Equation Problem, SIAM J. Computation, 15 (1986) $903-918$. DOI: $10.1137 / 0215063$

[7] F. Dumortier, J. Llibre, J. C. Artés, Qualitative theory of planar polynomial systems, Springer, Berlin 2006.

[8] X. Gómez-Mont, L. Ortíz-Bobadilla, Sistemas dinámicos holomorfos en superficies, Sociedad Matemática Mexicana, México D.F., 1989.

[9] J.E. Humphreys, Linear Algebraic Groups, Springer Verlag, New York, 1981.

[10] Y. Ilyashenko, S. Yakovenko, Lectures on Analytic Differential Equations, American Mathematical Society, Rhode Island, 2008.

[11] E. Kaltofen, A note on the Risch differential equation. in Proceedings of EUROSAM 84, Cambridge, England, July 9-11, 1984, Ed. by J. Fitch, pp. 359-366. DOI: 10.1007/BFb0032858

[12] E. R. Kolchin, Algebraic Matric Groups and the Picard-Vessiot Theory of Homogeneous Linear Ordinary Differential Equations, Annals of Mathematics 49 (1948) 1-42.

[13] E. R. Kolchin, Differential Algebra and Algebraic Groups, Academic Press, New York 1973.

[14] J. Liouville, Mémoire sur l'intégration d'une classe de fonctions transcendentes, Journal für ddie reine und angewandte Mathematik , 13 (1835) 93-118.

[15] J. Martinet, J.P. Ramis, Théorie de Galois différentielle et resommation, Computer Algebra and Differential Equations, E. Tournier, Ed. Academic Press, London (1989) 117-214.

[16] Juan J. Morales-Ruiz, Picard-Vessiot theory and integrability, Journal of Geometry and Physics $\mathbf{8 7}$ (2015) 314-343. DOI: 10.1016/j.geomphys.2014.07.006

[17] J.J. Morales-Ruiz, Differential Galois Theory and Non-integrability of Hamiltonian Systems, Progress in Mathematics 179, Birkhäuser, Basel 1999.

[18] J.J. Morales-Ruiz, J.P. Ramis, C. Simó, Integrability of Hamiltonian Systems and Differential Galois Groups of Higher Variational Equations, Ann. Sc. École Norm. Sup. 40 (2007) 845-884. DOI: 10.1016/j.ansens.2007.09.002

[19] M.J. Prelle, M. F. Singer, Elementary first integrals of differential equations, Trans. Amer. Math. Soc. 279 (1983) 215-229.

[20] R.H. Risch, The Problem of Integration in Finite Terms, Trans. Amer. Math. Soc. 139 (1969) 167-189.

[21] J.F. Ritt, Integration in Finite Terms, Columbia Univ. Press, New York 1948.

[22] M. Rothstein, Aspects of Symbolic Integration and Simplification of Exponential and Primitive Functions, Ph.D. thesis, Univ. Wisconsin-Madison, 1976.

[23] M.F. Singer, Liouvillian first integrals of differential equations, Trans. Amer. Math. Soc. 333 (1992), no. 2, 673-688.

[24] M. van der Put and M. Singer, Galois Theory of linear differential equations, Springer Verlag, Berlin, 2003.

[25] W. Wasow, Asymptotic expansions for ordinary differential equations, Dover, New York, 1965.

[26] E. T. Whittaker, An expression of certain known functions as generalised hypergeometric functions, Bull. Amer. Math. Soc. 10 (1903) 125-134.

[27] X. Zhang, Liouvillian integrability of polynomial differential systems, Trans. Amer. Math. Soc. 368 (2016) 607-620.

[28] X. Zhang, Integrability of Dynamical Systems: Algebra and Analysis, Developments in Mathematics, 47, Springer, Singapore, 2017. 
(P. Acosta-Humánez) Facultad de Ciencias Básicas y Biomédicas, Universidad Simón Bolívar, BARRANQUilla - COlOMBia

E-mail address: primitivo.acosta@unisimonbolivar.edu.co -- primi@intelectual.co

(J. T. Lázaro) Departament de Matemàtiques, Universitat Politècnica de Catalunya, Spain. E-mail address: jose.tomas.lazaro@upc.edu

(J.J. Morales-Ruiz) Departamento de Matemática Aplicada, Universidad Politécnica de Madrid, SPAIN.

E-mail address: juan.morales-ruiz@upm.es

(Ch. Pantazi) Departament de Matemàtiques, Universitat Politècnica de Catalunya, Spain.

E-mail address: chara.pantazi@upc.edu 